Revue de droit comparé du travail et de la sécurité sociale

2| 2019

L'ubérisation du travail

\title{
La jurisprudence sur l'ubérisation du travail au Royaume-Uni
}

\section{Lisa Rodgers}

\section{OpenEdition}

1 Journals

\section{Édition électronique}

URL : https://journals.openedition.org/rdctss/1553

DOI : 10.4000/rdctss. 1553

ISSN : 2262-9815

Éditeur

Centre de droit comparé du travail et de la sécurité sociale

\section{Édition imprimée}

Date de publication : 1 juin 2019

Pagination : 40-47

ISSN : 2117-4350

\section{Référence électronique}

Lisa Rodgers, "La jurisprudence sur l'ubérisation du travail au Royaume-Uni », Revue de droit comparé du travail et de la sécurité sociale [En ligne], 2 | 2019, mis en ligne le 01 novembre 2021, consulté le 11 novembre 2021. URL : http://journals.openedition.org/rdctss/1553 ; DOI : https://doi.org/10.4000/ rdctss. 1553

\section{(c) (†) $९$}

Revue de droit comparé du travail et de la sécurité sociale est mise à disposition selon les termes de la Licence Creative Commons Attribution - Pas d'Utilisation Commerciale - Pas de Modification 4.0 International. 


\section{LA JURISPRUDENCE SUR L'UBÉRISATION DU TRAVAIL AU ROYAUME-UNI}

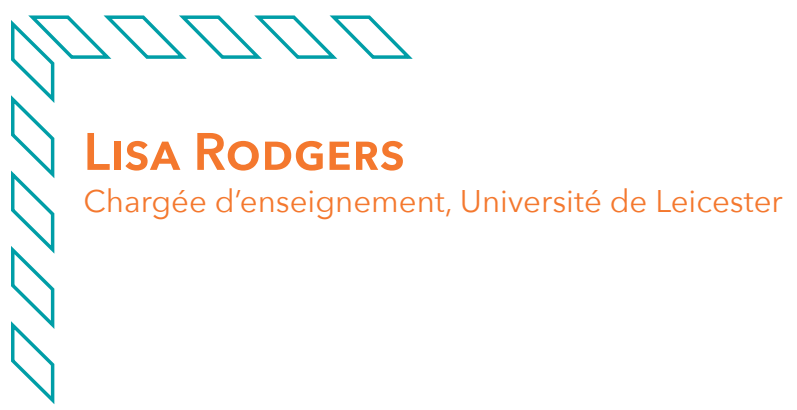

émergence et l'essor de la " gig economy" ", et notamment le recours aux plateformes Internet pour « recruter » des travailleurs afin de fournir des services, posent un certain nombre de problèmes concernant la détermination des droits du travail au Royaume-Uni comme ailleurs. Dans ce pays, la détermination des droits en matière d'emploi dépend de la distinction entre "salarié ", " travailleur » et " travailleur indépendant »; les salariés bénéficiant des droits les plus étendus en matière d'emploi, les travailleurs indépendants en ayant le moins. La détermination du statut est effectuée selon une série de critères factuels, qui se chevauchent dans une certaine mesure. En tout état de cause, en raison de la complexité des schémas d'organisation du travail au sein de la " gig economy », il s'avère très difficile de déterminer le statut de ces travailleurs. En réalité, ce type d'emploi présente des caractéristiques communes aux différents statuts. Par exemple, d'une part les travailleurs de la " gig economy » peuvent bénéficier d'une grande autonomie dans certains aspects de leur travail - ce qui suggère un statut de travailleur indépendantalors que, d'autre part, il peut exister un degré de contrôle élevé dans d'autres domaines d'activité, indiquant plutôt une relation salarié-employeur entre la plateforme et le prestataire de services. Cela constitue le premier ensemble de défis que présente la "gig economy ».

Une deuxième série de difficultés découle de l'action des sociétés plateformes qui tentent de dissimuler la « vraie » nature des relations entre les parties. Dans

1 La " gig economy » peut s'étendre à différents modèles d'activité. Pour une discussion de ces modèles dans le contexte de l'emploi, voir A. Todoli-Signes, "The End of the Subordinate Worker? The On-Demand Economy, the Gig Economy and the Need for Protection for Crowdworkers », Revue internationale de droit comparé du travail et des relations professionnelles, 2018, 33(2), p.241. 
un certain nombre d'affaires portées devant les tribunaux britanniques, la présentation de la relation entre la plateforme et le travailleur de la plateforme a été remise en question. A plusieurs reprises, il a été constaté que les conditions écrites du contrat entre les parties ne reflétaient pas la réalité de la relation de travail. En effet, dans l'affaire Uber, les tribunaux se sont montrés très critiques au sujet des actions menées par la plateforme pour tenter de dissimuler la véritable nature de la relation. Le tribunal a ainsi jugé qu'Uber avait déployé des efforts considérables pour donner une idée trompeuse de ces relations, " « en s'appuyant sur des fictions, sur un langage tordu et même sur une toute nouvelle terminologie », dans le but d'éviter que l'on juge que l'entreprise avait l'obligation d'accorder des droits aux travailleurs de la plateforme ${ }^{2}$. Ironiquement, il a été jugé que cela ne faisait que renforcer le poids de l'argument selon lequel les travailleurs étaient dépendants et n'avaient que très peu de pouvoir de négociation dans la relation.

Le troisième défi relève d'une étude conceptuelle plus approfondie sur la question de savoir si, et dans quelle mesure, les statuts actuels sont adaptés à la protection des travailleurs vulnérables. Par exemple, le manque de protection des travailleurs indépendants dans le système actuel s'avère particulièrement problématique dans le contexte de la " gig economy ", le statut de "travailleur indépendant» (même artificiel) pouvant dans ces circonstances être source de vulnérabilité. Au Royaume-Uni, il existe des interrogations relatives à la valeur de la distinction entre les statuts de "travailleur » et de "salarié ", les travailleurs vulnérables des plateformes retenant le statut de « travailleur » comme stratégie procédurale, mais sous-évaluant souvent leur vulnérabilité et donc les droits qui leur sont dus.

Dans cette contribution, nous aborderons les deux premières séries de difficultés, tout en notant que des discussions conceptuelles plus approfondies sur l'adéquation des différents statuts semblent inévitables. La première partie présente le régime légal de droit commun qui détermine la délimitation du statut professionnel au Royaume-Uni (I). La deuxième identifie certains éléments problématiques dans ces définitions s'agissant de leur application aux travailleurs de la " gig economy» (II). Comme les affaires concernent le statut de " travailleur», les éléments problématiques de ce statut seront examinés: exécution personnelle, « réciprocité des obligations » et existence d'un lien contractuel entre l'employeur et le salarié. Cette partie illustre également les manipulations possibles de ces éléments par des employeurs puissants (tels qu'Uber) pour tenter d'influencer le résultat des différents litiges. En conclusion, on présentera les propositions du gouvernement du Royaume-Uni pour améliorer le statut des travailleurs de la " gig economy ».

\section{I - LES STATUTS D'EMPLOI : SALARIÉ, TRAVAILLEUR, TRAVAILLEUR INDÉPENDANT}

Pendant de longues années, la détermination du statut professionnel au Royaume-Uni a été basée sur une division binaire entre le statut de salarié et le statut d'indépendant. Cependant, un statut intermédiaire de travailleur a été intégré dans les lois à la fin des années 90, afin d'étendre un ensemble de droits « fondamentaux » à un plus grand nombre de personnes et d'atténuer les effets d'une division binaire perçue comme trop rigide. Ainsi, il existe désormais au Royaume-Uni trois catégories de statut d'emploi : "salarié ", " travailleur » et " travailleur indépendant ». Chacune de ces catégories implique un 
ensemble distinct de droits du travail. Les « salariés » ont droit à l'intégralité des droits liés à l'emploi, dont le droit de recours contre le licenciement abusif ${ }^{3}$ et la protection contre le transfert du contrat de travail ${ }^{4}$. Tel n'est pas le cas des travailleurs qui bénéficient d'un nombre limité de protections, dont les droits consacrés par le Règlement sur le temps de travail 5 et par la Loi sur le salaire minimum national ${ }^{6}$. Les «travailleurs indépendants " disposent de droits très limités, mais bénéficient de la protection de la santé et de la sécurité au travail7, ainsi que des droits à l'égalité ${ }^{8}$.

L'article 230 (1) de la Loi de 1996 sur les droits du travail énonce le critère légal pour déterminer le statut de salarié. II prévoit qu'il s'agit des personnes qui travaillent sous " contrat de travail », à savoir " un contrat de service ou d'apprentissage, explicite ou implicite ». Ce critère légal ayant un caractère plutôt général et superficiel, les tribunaux britanniques ont mis au point une série de critères supplémentaires afin de déterminer si une personne est salariée ou non. La popularité de ces critères a fluctué selon les époques. Vers 1900, c'était la question du « contrôle » qui était invoquée pour déterminer le statut de salarié, mais celle-ci a perdu de sa popularité au cours de la première moitié du XXème siècle, laissant place à d'autres critères. Le critère du contrôle a ensuite connu une résurgence avec l'affaire Ready Mixed Concrete en 1968\%, qui suggérait que plusieurs critères soient associés pour déterminer le statut professionnel, dont celui de la question du contrôle. Si l'on continue à évoquer cette «multiplicité de critères », c'est la question de la réciprocité des obligations qui domine aujourd'hui la détermination du statut de salarié. Cette "réciprocité » a été interprétée de différentes manières. La notion fait référence à l'affirmation assez simple selon laquelle il doit exister une prestation de travail en échange d'une rémunération pour qu'il y ait un contrat de travail (c'est la signification qui en a été donnée dans l'affaire Ready Mix). Cependant, la tendance jurisprudentielle a été d'introduire une notion d'obligation dans ce concept. En conséquence, la notion de réciprocité des obligations a évolué de sorte que sont intégrées une certaine continuité, stabilité et relation humaine dans le contrat de travail ${ }^{10}$. La réciprocité, dans ce sens, dépend de la question de savoir s'il existe une obligation pour l'employeur de fournir du travail et une obligation corrélative pour le salarié de l'exécuter ${ }^{11}$.

En pratique, cette "réciprocité des obligations » est extrêmement difficile à établir, en particulier pour les groupes de "travailleurs atypiques » dont les contrats réfutent explicitement l'existence de ce type de relation. C'est le cas notamment des travailleurs de la "gig economy", dont les contrats sont spécifiquement conçus pour être flexibles, sans engagement continu de la part de «l'employeur » de fournir du travail ni de son acceptation par le travailleur de la plateforme. C'est en partie pour cette raison que les travailleurs de la

3 Employment Rights Act, 1996, Section 94.

4 Transfer of Undertakings Protection of Employment Regulations, 2006.

5 Working Time Regulations, 1998, Regulation 2 (1).

6 National Minimum Wage Act, section 54.

7 Health and Safety at Work Act, section 53.

8 Equality Act, 2010, section 83 (2).

9 Ready Mixed Concrete $v$ Minister of Pensions and National Insurance [1968] 2 OB 497.

10 N. Countouris, "Uses and Abuses of 'Mutuality of Obligations' and the Autonomy of Labour Law " in A. Bogg, C. Costello, A. CL Davies and J. Prassl, The Autonomy of Labour Law, Oxford Hart Publishing, 2015, p.187.

11 Nethermere v Gardiner [1984] ICR 612 at 632. 
plateforme n'ont pas cherché à revendiquer le statut de salariés, mais se sont contentés de demander le statut de «travailleur ». Cependant, à certains égards, les critères permettant de prouver le statut de travailleur sont encore plus vagues et confus que ceux relatifs au statut de salarié. En effet, certaines affaires ayant fait jurisprudence suggèrent même que la réciprocité des obligations est nécessaire, non seulement pour prouver le statut de salarié, mais également pour attester celui de travailleur. Cette affirmation est sujette à controverse, notamment au regard de la version la plus complète de la définition ${ }^{12}$.

La définition légale du travailleur est énoncée à l'article 230 (3) de la Loi sur les droits du travail, qui dispose qu'un travailleur est une personne qui travaille en vertu d'un contrat de travail ou de " tout autre contrat, explicite ou implicite et [s'il est explicite], sous forme orale ou écrite, et en vertu duquel elle s'engage à exécuter ou effectuer personnellement un travail ou des services pour une autre partie au contrat, dont le statut n'est pas en vertu du contrat celui d'un client ou d'une entreprise commerciale exercée par un particulier ». Cette définition comporte deux caractéristiques. Tout d'abord, il doit exister un " contrat » ou un lien « contractuel». Cela implique que la relation entre l'individu et l'entreprise ne doit pas être celle d'un client, car une telle relation démontrerait l'existence d'un niveau d'indépendance du travailleur plus compatible avec le travail indépendant qu'avec le statut de travailleur. Par ailleurs, il doit exister un degré de «service personnel » de la part de la personne fournissant les services ${ }^{13}$.

\section{II - LE STATUT DE TRAVAILLEUR ET LE LIEN CONTRACTUEL}

Au Royaume-Uni, l'une des premières affaires relative à la question du statut professionnel dans la "gig economy " a été l'affaire Aslam v Uber ${ }^{14}$. Les demandeurs étaient des chauffeurs d'Uber. Ils affirmaient qu'ils s'étaient vu refuser le paiement de leur indemnité de congés payés et qu'Uber ne leur avait également pas versé le salaire minimum national. Ces deux plaintes reposaient sur l'établissement de la preuve du statut de "travailleur ».Le tribunal de première instance a donc décidé de déterminer si les chauffeurs d'Uber appartenaient ou non à cette catégorie. En octobre 2016, le tribunal de première instance a jugé que les conducteurs répondaient à la définition de " travailleur " au sens de l'article 230 (3) b) de la Loi sur les droits du travail. Par la suite, Uber a fait appel du jugement au motif que le tribunal s'était trompé sur la nature de sa relation avec les chauffeurs, arguant que ces derniers étaient en fait des "travailleurs indépendants ». L'audience en appel a eu lieu en novembre $2017^{15}$. Un autre recours a ensuite été formé devant la Cour d'appel. L'arrêt de la Cour d'appel a été rendu le 19 décembre $2018^{16}$. Le tribunal d'appel en matière du travail et la Cour d'appel ont confirmé en grande partie les conclusions initiales du tribunal de première instance.

En appel, la principale question qui devait être tranchée était celle de savoir s'il existait ou non un « contrat » ou un « lien contractuel » avec la société locale d'Uber (Uber London-ULL) afin d'établir le statut de travailleur. Les représentants d'ULL affirmaient que les chauffeurs d'Uber n'avaient jamais travaillé sous contrat avec eux. En réalité, ULL agissait

12 J. Prassl, «Who is a Worker », 2017, 133 LQR 366, p.371.

13 D. Cabrelli.

14 [2017] IRLR 4.

15 [2018] ICR 453.

16 [2018] EWCA Civ 2748. 
simplement en qualité de mandataire des chauffeurs, dans la fourniture de services de transport aux passagers. ULL a mis en avant le libellé de la documentation contractuelle régissant la relation entre Uber et les conducteurs, qui était présentée comme une relation de mandat et énonçait les conditions de cette relation censée être compatible avec cette qualité ${ }^{17}$. Les conducteurs ont contesté cette interprétation de la relation. Ils ont affirmé avoir personnellement travaillé pour ULL dans le cadre de leur activité, celle-ci consistant à fournir des services de transport aux passagers de la région de Londres.

La Cour d'appel a confirmé que, pour que les chauffeurs d'Uber satisfassent à la définition de travailleur conformément à l'alinéa b) de l'article 230 (3) de la Loi sur les droits du travail, il serait nécessaire de démontrer qu'il existait en réalité un « contrat » entre les deux parties à la relation. Ce contrat pouvait être purement basé sur des missions, exécutées si et quand un travail était entrepris, mais un lien contractuel devait exister. Toutefois, l'approche retenue par la Cour d'appel en la matière consistait à déterminer le « véritable accord » existant entre les parties, conformément au jugement de la Cour suprême dans l'affaire Autoclenz v Belcher ${ }^{18}$. Cela nécessitait d'examiner la « réalité de la relation » qui pouvait ou non être reflétée précisément dans les documents contractuels eux-mêmes ${ }^{19}$. En effet, Autoclenz avait spécifiquement reconnu le risque inhérent à l'utilisation de documents contractuels écrits dans le cadre de relations de travail caractérisées par une inégalité du pouvoir de négociation entre les parties. Il incombait donc à la Cour d'appel de rechercher l'intention des parties pour décider si les termes d'un accord écrit reflétaient les véritables intentions des parties ${ }^{20}$. En adoptant cette approche, la Cour d'appel a décidé que la documentation contractuelle entre ULL et les conducteurs ne reflétait pas la réalité de la relation. Conformément aux conclusions du tribunal de première instance, elle a jugé que, dans la pratique, la relation entre ULL et les conducteurs n'était pas celle d'un mandataire et d'un mandant, dans laquelle les conducteurs auraient entretenu une relation contractuelle directe avec les passagers. En effet, il ressortait des éléments de preuve que les chauffeurs n'étaient pas en mesure d'établir une relation d'affaires avec les passagers, car ils ne pouvaient ni obtenir leurs coordonnées, ni fournir les leurs. Au lieu de cela, les chauffeurs étaient des travailleurs sous contrat avec ULL. Ils étaient intégrés dans l'activité d'Uber, consistant à fournir des services de transport commercialisés en tant que tels. Ils travaillaient en partant du principe qu'Uber les indemniserait pour les impayés, et étaient soumis à divers contrôles dans l'exercice de leurs fonctions, bien que ces contrôles s'appliquent « indirectement ». Par exemple, même si un chauffeur avait la " possibilité » d'annuler un travail une fois accepté, Uber infligeait un avertissement à ceux qui annulaient une course de cette manière.

La Cour d'appel s'est ensuite penchée sur la question de l'étendue et du calendrier des obligations contractuelles. Bien qu'elle ait conclu que les chauffeurs d'Uber se trouvaient dans une relation contractuelle directe " quand ils étaient sur le terrain, avec l'application activée et prête à accepter des missions ", la Cour d'appel a fait preuve de plus de

17 Uber (EAT) (n 15), para 91.

18 [2011] ICR 1157.

19 Uber (CA) (n 16), para 77.

20 II est intéressant de noter que la Cour a également été influencée dans cette approche par la reconnaissance de l'objet légal des dispositions en cause dans la présente affaire, qui reconnaissaient expressément la nécessité de protéger les parties les plus vulnérables de la relation de travail Uber (EAT) (n 15), paragraphe 98. 
circonspection sur la relation entre Uber et le travailleur en dehors des missions. Cependant, elle a conclu que le tribunal de première instance avait eu raison de juger, compte tenu des faits, qu'il existait suffisamment de réciprocité d'obligation entre les missions pour que le contrat perdure après l'exécution de celles-ci ${ }^{21}$. En fait, cette « réciprocité des obligations » a été évoquée dans d'autres affaires concernant des travailleurs de la " gig economy ». Dans Addison Lee par exemple, la Cour a constaté que, malgré l'absence de preuve écrite d'un ensemble d'obligations globales entre la société de plateforme et les livreurs, ces obligations existaient bel et bien dans la pratique ${ }^{22}$. La reconnaissance de l'existence d'une telle obligation réciproque est importante car elle montre à quel point certains de ces travailleurs se rapprochent des modèles «traditionnels " d'obligation et combien la documentation contractuelle peut induire en erreur. D'un autre côté, l'introduction de ce type d'exigence de réciprocité dans la détermination du statut de travailleur présente des risques ${ }^{23}$. Il est clair qu'un grand nombre de personnes travaillant au sein de la "gig economy » ne seraient pas en mesure de prouver cette "réciprocité », ce qui irait à l'encontre de l'intention du Parlement de vouloir étendre la protection à travers la définition du travailleur.

\section{III - LE SERVICE PERSONNEL}

Le deuxième élément majeur pour prouver le statut de travailleur visé à l'alinéa (b) concerne le service réalisé à titre personnel. La nécessité de prouver l'existence d'un service personnel est spécifiquement mentionnée dans l'alinéa (b) et a été citée dans la jurisprudence comme une condition nécessaire à l'établissement du statut de travailleur ${ }^{24}$. Cette question ne s'est pas posée dans l'affaire Uber car, dans le modèle Uber, le travail est supposé être effectué en personne et il existe des pénalités en cas de non-respect de cette obligation. Cependant, la preuve du service personnel a été une question importante dans d'autres affaires relatives à des travailleurs de la "gig economy ». La manière de traiter les «clauses de substitution » dans les contrats de travail a été un problème particulièrement épineux. Ces clauses de substitution visent à permettre aux travailleurs de substituer leur propre travail à celui d'un autre, et leur existence peut donc être invoquée pour suggérer l'absence d'une obligation de service personnel entre l'individu et la plateforme.

Cette argumentation particulière a été employée avec succès par la plateforme Roofoods Limited (exerçant ses activités sous le nom de Deliveroo), pour affirmer ainsi que les coursiers de Deliveroo n'étaient pas soumis à l'obligation de prester personnellement le travail requis par la loi et nécessaire à l'établissement du statut de travailleurs. Cette affaire diffère de celle d'Uber en ce qu'elle a été introduite par un syndicat, I'Union indépendante des travailleurs de Grande-Bretagne (IWGB), et non par les travailleurs. Ce syndicat a demandé la reconnaissance de la qualité de syndicat représentant des conducteurs de Deliveroo (encore faut-il que les chauffeurs de Deliveroo soient des «travailleurs » au sens de l'article 296 de la Loi de 1992 sur les syndicats et les relations de travail (loi consolidée -TULRCA). L'affaire a été entendue par le Comité central d'arbitrage en novembre 2017. Le

21 Uber (EAT) (n 15), para 121.

22 Addison Lee Limited v Mr M. Lange, Mr M. Olszewski, Mr M. Morahan [2018] WLR 05929292.

23 J. Prassl (n 12) 371.

24 Pimlico Plumbers Limited v Smith [2018] ICR 1511. 
syndicat a ensuite fait appel devant la Queen's Bench Division ${ }^{25}$. L'affaire fut alors portée devant la Haute Cour. Le jugement a été rendu en décembre $2018^{26}$.

La Haute Cour a souligné l'importance de prouver le caractère intuitu personae de la relation pour conclure que les personnes étaient des travailleurs au sens de l'article 296 de la TULRCA. La Cour s'est référée spécifiquement à l'arrêt de la Cour suprême dans l'affaire Pimlico Plumbers ${ }^{27}$, dans lequel il était indiqué que, s'il existait un « droit de substitution généralisé ", il ne pouvait y avoir de service personnel et donc pas de statut de travailleur ${ }^{28}$. La Cour s'est basée sur les conclusions du Comité central d'arbitrage au sujet de la nature factuelle des clauses de substitution figurant dans les contrats de Deliveroo. Elle a conclu que le droit de substitution était effectivement " généralisé ", puisqu'il était à la fois "sans entrave » et "véritable ». II était sans entrave, car très peu de conditions étaient imposées pour l'utilisation de substituts par Deliveroo. La seule condition réelle était que les coursiers de Deliveroo ne pouvaient pas faire appel à une personne précédemment engagée par Deliveroo et dont le contrat avait été résilié pour faute professionnelle par la société. En outre, la Haute Cour partageait la position du Comité central d'arbitrage sur le fait que le droit de substitution était "véritable », en ce sens qu'il était appliqué par des conducteurs dans la pratique. Ces deux éléments signifiaient que les conducteurs de Deliveroo ne pouvaient pas prouver qu'ils effectuaient un service à titre personnel et donc ne pouvaient pas être reconnus comme des travailleurs au sens de la loi.

Contrairement au jugement rendu dans l'affaire Deliveroo, il a été jugé dans d'autres affaires qu'un service personnel existait bel et bien, malgré l'existence d'une clause contractuelle de substitution. Cette distinction a été opérée de deux manières. Premièrement, la clause de substitution en question a été jugée comme étant extrêmement restrictive dans les faits, sujette à des conditions assez étendues ou rarement utilisées dans la pratique. Deuxièmement, les tribunaux ont été plus disposés à adopter une approche « intentionnelle » de la construction des clauses de substitution que la Haute Cour dans l'affaire Deliveroo ${ }^{29}$. En d'autres termes, ils ont cherché la véritable intention des parties. Bien entendu, ces deux voies peuvent être liées : une approche plus intentionnelle pourrait conduire à la conclusion qu'une clause de substitution est plus étroite, qu'elle n'apparaît à première vue et ne contredit donc pas une décision en faveur du statut de travailleur ${ }^{30}$. Tel a été le sens du jugement rendu dans l'affaire Pimlico, et il fut suivi dans d'autres affaires. Dans cette affaire, il a été jugé que le demandeur disposait d'un pouvoir limité de se faire remplacer par d'autres travailleurs. Cependant, la Cour suprême a approuvé la Cour d'appel sur le fait que ce droit de substitution n'entravait pas l'obligation de service personnel du demandeur. Au contraire, la « caractéristique dominante » du contrat restait le service personnel, ce qui était parfaitement en harmonie avec les autres stipulations contractuelles ${ }^{31}$.

25 Independent Workers Union of Great Britain v Roofoods Limited (t/a Deliveroo) [2018] EWHC 1939 (Admin).

26 Independent Workers Union of Great Britain v Roofoods Limited (t/a Deliveroo) [2018] EWHC 3342 (Admin).

27 [2018] ICR 1511.

28 Deliveroo (n 26) para 66.

29 La Haute Cour, dans l'affaire Deliveroo, a refusé de soutenir tout argument fondé sur Autoclenz. Voir Deliveroo (n 26), paragraphe 64.

30 Conformément au jugement dans l'affaire Macfarlane v Glasgow City Council [2001] IRLR 7.

31 Pimlico Plumbers v Smith [2018] UKSC 29, paragraphe 33. 
Cette décision a été suivie dans l'affaire Leyland $v$ Hermes $^{32}$. Dans cette affaire, le service personnel a également été considéré comme une « caractéristique dominante » du contrat malgré l'existence d'une clause de substitution. Cela a été prouvé par le fait que, même s'il était possible de nommer un remplaçant un jour donné, le «travailleur » restait responsable de la nomination et devait communiquer avec la direction au sujet du remplacement ou superviser le remplaçant. En conséquence, le contrat exigeait « qu'une partie au moins des tâches soit effectuée par le coursier lui-même $»^{33}$.

\section{CONCLUSION}

Les affaires concernant la "gig economy » ont clairement mis en lumière les problèmes liés à la détermination des statuts en droit du travail britannique. Il semble que l'obtention du statut de "salarié " soit si difficile, que cette stratégie est rarement adoptée dans les affaires où l'on cherche à faire respecter ses droits en tant que travailleur. Ceci en dépit des preuves que la réciprocité peut souvent exister dans un large éventail de contrats, au sein de la "gig economy » et en dehors. En ce qui concerne le statut de travailleur, il existe une incohérence considérable dans la jurisprudence, qui s'explique par un certain nombre de raisons. D'une part, la définition dans les textes est relativement vague et formulée en termes négatifs. D'autre part, il existe une tendance à estomper les frontières entre le statut de salarié et celui de travailleur, comme cela a été démontré à propos du critère de la réciprocité des obligations dans la définition du travailleur. Enfin, la " gig economy » engendre des schémas factuels et des arrangements contractuels extrêmement complexes, qui peuvent être délibérément conçus pour écarter le statut de travailleur, et ceux-ci doivent être minutieusement examinés par les tribunaux. Au final, les litiges exacerbent la tension séculaire du droit du travail entre le respect de l'accord " conclu » entre les parties et la reconnaissance du déséquilibre dans le pouvoir de négociation entre les parties qui ternit l'accord conclu. Les propositions de réforme du statut de l'emploi au Royaume-Uni, actuellement examinées par le gouvernement, ne traitent pas de cette tension fondamentale et n'ont donc qu'un impact limité sur l'amélioration de la condition des travailleurs au sein de la "gig economy $»^{34}$.

32 Ms E. Leyland and others v Hermes Parcelnet [2018] WLR 03145778.

$33 \mathrm{Ibid}$, paragraphe 79.

34 M. Taylor, « Good Work: The Taylor Review of Modern Working Practices » (Juillet 2017). 